\title{
The plasmid-mediated evolution of the mycobacterial ESX (Type VII) secretion systems
}

\author{
Mae Newton-Foot ${ }^{*}$ (D, Robin Mark Warren, Samantha Leigh Sampson, Paul David van Helden \\ and Nicolaas Claudius Gey van Pittius
}

\begin{abstract}
Background: The genome of Mycobacterium tuberculosis contains five copies of the ESX gene cluster, each encoding a dedicated protein secretion system. These ESX secretion systems have been defined as a novel Type VII secretion machinery, responsible for the secretion of proteins across the characteristic outer mycomembrane of the mycobacteria. Some of these secretion systems are involved in virulence and survival in M. tuberculosis; however they are also present in other non-pathogenic mycobacteria, and have been identified in some non-mycobacterial actinomycetes. Three components of the ESX gene cluster have also been found clustered in some gram positive monoderm organisms and are predicted to have preceded the ESX gene cluster.
\end{abstract}

Results: This study used in silico and phylogenetic analyses to describe the evolution of the ESX gene cluster from the WXG-FtsK cluster of monoderm bacteria to the five ESX clusters present in M. tuberculosis and other slowgrowing mycobacteria. The ancestral gene cluster, ESX-4, was identified in several nonmycomembrane producing actinobacteria as well as the mycomembrane-containing Corynebacteriales in which the ESX cluster began to evolve and diversify. A novel ESX gene cluster, ESX-4EVoL, was identified in some non-mycobacterial actinomycetes and $M$. abscessus subsp. bolletii. ESX-4 EVOL contains all of the conserved components of the ESX gene cluster and appears to be a precursor of the mycobacterial ESX duplications. Between two and seven ESX gene clusters were identified in each mycobacterial species, with ESX-2 and ESX-5 specifically associated with the slow growers. The order of ESX duplication in the mycobacteria is redefined as ESX-4, ESX-3, ESX-1 and then ESX-2 and ESX-5. Plasmid-encoded precursor ESX gene clusters were identified for each of the genomic ESX-3, $-1,-2$ and -5 gene clusters, suggesting a novel plasmid-mediated mechanism of ESX duplication and evolution.

Conclusions: The influence of the various ESX gene clusters on vital biological and virulence-related functions has clearly influenced the diversification and success of the various mycobacterial species, and their evolution from the non-pathogenic fast-growing saprophytic to the slow-growing pathogenic organisms.

Keywords: ESX, ESAT-6, Evolution, Mycobacterium, Plasmid, Type VII secretion system

\section{Background}

The genome of Mycobacterium tuberculosis contains five ESX (or ESAT-6) gene clusters, named ESX-1, -2, -3, -4 and -5 , which encode the Esx and PE/PPE proteins, various ATPases, membrane proteins, the mycosin proteases and other ESX-associated proteins [1,2]. The ESX gene

\footnotetext{
* Correspondence: maen@sun.ac.za

Division of Molecular Biology and Human Genetics, Department of Biomedical Sciences, DST/NRF Centre of Excellence for Biomedical Tuberculosis Research/ SAMRC Centre for Molecular and Cellular Biology, Faculty of Medicine and

Health Sciences, Stellenbosch University, Stellenbosch, South Africa
}

clusters have been the topic of extensive research following the discovery that the primary attenuating deletion of $M$. bovis BCG, region of difference 1 (RD1), includes part of ESX-1 [3-5]. The proteins encoded in each of the ESX gene clusters have been predicted to form dedicated protein secretion systems, the ESX secretion systems, which have since been defined as a Type VII secretion machinery responsible for the secretion of, amongst others, the Esx, PE and PPE proteins encoded in them, across the outer mycomembrane $[6,7]$. 
The functions of the five M. tuberculosis ESX secretion systems appear to be distinct. ESX-1 is associated with virulence in $M$. tuberculosis [8-10], where it is involved in the inhibition of T-cell responses and phagosome maturation [11, 12], and assists in the escape of mycobacteria from the macrophage vacuole by ESAT-6-mediated perforation of the vacuolar membrane [13-16]. ESX-5 has also been linked to $M$. tuberculosis pathogenicity and is involved in modulating the host immune responses to maintain a persistent infection $[15,17,18]$. ESX-5 has furthermore been linked to the uptake of nutrients by increasing outer-membrane permeability in the slowgrowing mycobacteria [19]. ESX-3 is essential for the in vitro growth of $M$. tuberculosis [20, 21], and is involved in divalent cation (iron and zinc) homeostasis [22, 23], and specifically iron uptake via the mycobactin iron acquisition pathway [21, 24]. The functions of ESX-2 and ESX-4 remain unknown.

The ESX gene clusters occur throughout the genus Mycobacterium. A previous study has proposed the order of duplication of the ESX gene clusters to be ESX-4, -1, -3, -2 and then -5 , with ESX-5 exclusively associated with the slow-growing mycobacteria [2]. The non-pathogenic, fastgrowing mycobacterium, $M$. smegmatis, contains three of the five $M$. tuberculosis ESX gene clusters, ESX-1, -3 and -4 [2]. In $M$. smegmatis, ESX-1 has been shown to be involved in conjugal DNA transfer $[25,26]$. ESX-3 is also involved in iron homeostasis, however it has not been directly linked to zinc homeostasis, and is not essential in this organism [27]. Although there are distinct contrasts in the functions of these secretion systems in M. smegmatis and M. tuberculosis, the orthologous systems have been shown to share certain characteristics and to secrete both sets of substrates $[25,28,29]$. This suggests that the ESX secretion systems have retained conserved mechanisms, and that virulence-associated functions may have evolved subsequently, or be associated with specific substrates.

ESX gene clusters have also been identified in the genomes of closely related actinomycetes outside of the genus Mycobacterium, including Nocardia, Streptomyces and Corynebacteria [2, 6]. Furthermore, genes encoding two components of the ESX secretion system, the WXG (or Esx-like) and FtsK/SpoIIIE proteins, have been found clustered in some gram-positive monoderm genera such as Bacillus, Listeria and Saccharomyces [30]. Indeed, it has been suggested that ESX secretion systems occur outside of the Mycolata (species containing a mycomembrane-like outer membrane containing mycolic acids, including Corynebacteria, Rhodococci, Nocardia and Mycobacteria) and are therefore not typically involved in transmycomembrane secretion [31]. This, together with the absence of an identifiable component responsible for mycomembrane translocation, or an elucidated Type VII secretory mechanism, has generated some controversy, as some suggest that these are requirements for the designation of the ESX secretion systems as distinct Type VII secretion machineries [32].

Here we investigated the presence and absence of the ESX gene clusters in the genomes of the sequenced mycobacteria and other representative species from the class Actinobacteria. The phylogenetic relationship between these and the identified WXG-FtsK clusters of certain monoderm bacteria was determined in order to define the evolutionary history of the Type VII ESX secretion systems. In addition to the five ESX gene clusters which were previously identified, ESX gene clusters were identified on plasmids within several species of mycobacterium, and shown to precede the genomic ESX duplications. A model is proposed for the plasmidmediated duplication and evolution of the ESX gene clusters.

\section{Results and discussion}

ESX gene clusters were identified from the publicly available genome sequences of 60 actinobacterial species, including 40 mycobacterial species, 11 other species from the order Corynebacteriales and 9 species selected from the orders Pseudonocardiales, Glycomycetales, Micromonosporales, Frankiales, Streptosporangiales, Catenulisporales, Streptomycetales, Propionibacteriales and Kineosporiales (Table 1). Each genome contains between one and seven ESX gene clusters. The components and arrangement of each ESX gene cluster were determined and are represented in Additional file 1 with three WXG-FtsK clusters from Staphylococcus aureus, Listeria monocytogenes and Bacillus subtilis, identified in the literature as precursors of the ESX gene cluster [30,31]. The concatenated protein sequences of each ESX gene cluster were aligned and used to generate a phylogeny of the ESX gene clusters using maximum likelihood (ML) and distance methods (Fig. 1 and Additional file 2) using the WXG-FtsK clusters of $S$. aureus, L. monocytogenes and B. subtilis as the outgroup. The topology of the trees generated by ML and distance methods was conserved, depicting 5 distinct clades, each incorporating one of the M. tuberculosis H37Rv ESX gene cluster regions 1 to 5 .

ESX gene clusters were identified on plasmids in several mycobacterial species (pMFLV01 in M. gilvum, pMKMS01 and pMKMS02 in M. sp. KMS, Plasmid01 in M. sp. MCS, pMYCCH.01 and pMYCCH.02 in M. chubuense, pMYCSM01, pMYCSM02 and pMYCSM03 in M. smegmatis JS623, Plasmid 2 in M. abscessus sp. bolletii and pMyong1 in M. yongonense). Four additional mycobacterial plasmid-encoded ESX gene clusters were previously identified by Ummels et al., (2014) [33]. The sequences of three of these, on pRAW from $M$. marinum E11, pMAH135 from M. avium subsp. hominis suis T135 and pMK12478 from M. kansasii ATCC12478, are 
Table 1 WXG-FtsK and ESX gene clusters identified in sequenced mycobacterial and selected actinobacterial species

\begin{tabular}{|c|c|c|c|c|c|c|c|c|c|c|c|c|}
\hline \multirow[t]{2}{*}{ Species } & \multirow[t]{2}{*}{ WXG_FtsK } & \multicolumn{6}{|c|}{ Genomic ESX } & \multicolumn{5}{|c|}{ Plasmid ESX } \\
\hline & & 4 & $4 \mathrm{ev}$ & 3 & 1 & 2 & 5 & $\overline{\mathrm{P} 1}$ & P2 & P3 & $\mathrm{P}^{\prime}$ & P5 \\
\hline Bacillus subtilis subsp. subtilis str. 168 & $x$ & & & & & & & & & & & \\
\hline Catenulispora acidiphila DSM43021 & & $x x x x$ & & & & & & & & & & \\
\hline Corynebacterium diphtheriae NCTC 13129 & & $x$ & & & & & & & & & & \\
\hline Corynebacterium pseudotuberculosis FRC41 & & $x$ & & & & & & & & & & \\
\hline Frankia alni ACN14a & & $x$ & & & & & & & & & & \\
\hline Gordonia bronchialis DSM 43247 & & $x$ & & & & & & & & & & \\
\hline Janibacter sp. HTCC2649 & & $x$ & & & & & & & & & & \\
\hline Kribbella flavida DSM17836 & & $x x$ & & & & & & & & & & \\
\hline Listeria monocytogenes $\mathrm{L} 312$ & $x$ & & & & & & & & & & & \\
\hline M. abscessus & & $x$ & & $x$ & & & & & & & & \\
\hline M. abscessus subsp. bolletii 50594 & & & $x$ & $x$ & & & & & $x$ & & & \\
\hline M. africanum GM041182 & & $x$ & & $x$ & $x$ & $x$ & $x$ & & & & & \\
\hline M. avium 104 & & $x$ & & $x$ & & $x$ & $x$ & & & & & \\
\hline M. avium paratuberculosis K-10 & & $x$ & & $x$ & & $x$ & $x$ & & & & & \\
\hline M. bovis AF2122/97 & & $x$ & & $x$ & $x$ & $x$ & $x$ & & & & & \\
\hline M. bovis BCG Pasteur $1173 P 2$ & & $x$ & & $x$ & $x^{b}$ & $x$ & $x$ & & & & & \\
\hline M. canettii CIPT140010059 & & $x$ & & $x$ & $x$ & $x$ & $x$ & & & & & \\
\hline M. chubuense & & $x$ & & & $x$ & & & & & & $x x$ & \\
\hline M. colombiense CECT3035 ${ }^{\mathrm{a}}$ & & $\mathrm{x}$ & & $x$ & & $x$ & $x$ & & & & & \\
\hline M. fortuitum subsp. fortuitum DSM $46621^{\text {a }}$ & & $x$ & $x$ & $x$ & $x$ & & & & & & & \\
\hline M. gilvum PYR-GCK & & $x$ & & $x$ & $x$ & & & $x$ & & & & \\
\hline M. indicus pranii MTCC9506 & & $x$ & & $x$ & & $x$ & $x$ & & & & & \\
\hline M. intracellulare ATCC13950 & & $x$ & & $x$ & & $x$ & $x$ & & & & & \\
\hline M. kansasii ATCC12478 & & $x$ & & $x$ & $x$ & $x$ & $x$ & & & & & \\
\hline M. leprae TN & & & & $x$ & $x$ & & $x$ & & & & & \\
\hline M. ulcerans subsp. liflandii $128 \mathrm{FXT}$ & & $x$ & & $x$ & $x$ & & $x$ & & & & & \\
\hline M. marinum M & & $x$ & & $x$ & $x$ & & $x$ & & & & & \\
\hline M. massiliense CCUG48898 ${ }^{\mathrm{a}}$ & & $x$ & & $x$ & & & & & & & & \\
\hline M. microti $19422^{\mathrm{a}}$ & & $x$ & & $x$ & $x^{b}$ & $x$ & $x$ & & & & & \\
\hline M. neoaurum VKM Ac-1815D & & $x$ & & $x$ & $x$ & & & & & & & \\
\hline M. orygis 112400015 & & $x$ & & $x$ & $x$ & $x$ & $x$ & & & & & \\
\hline M. parascrofulaceum BAA-614 & & $x$ & & $x$ & & $x$ & $x$ & & $x$ & & $x$ & $x$ \\
\hline M. phlei RIVM601170 & & $x$ & & $x$ & $x$ & & & & & & & \\
\hline M. rhodesiae $\mathrm{NBB}^{\mathrm{a}}$ & & $x$ & & $x$ & $x$ & & & & & & & \\
\hline M. smegmatis $\mathrm{mc}^{2} 155$ & & $x$ & & $x$ & $x$ & & & & & & & \\
\hline M. sp. JDM601 & & $x$ & & $x$ & & $x$ & $x$ & & & & & \\
\hline M. sp. JLS & & $x$ & & $x$ & $x$ & & & & & & & \\
\hline M. sp. KMS & & $x$ & & $x$ & $x$ & & & & $x$ & $x$ & & \\
\hline M. sp. MCS & & $x$ & & $x$ & $x$ & & & & & $x$ & & \\
\hline M. sp. MOTT36Y & & $x$ & & $x$ & & $x$ & $x$ & & & & & \\
\hline M. sp. Spyr 1 & & $x$ & & $x$ & $x$ & & & & & & & \\
\hline M. thermoresistibile 19527 & & $x$ & & $x$ & $x$ & & & & & & & \\
\hline
\end{tabular}


Table 1 WXG-FtsK and ESX gene clusters identified in sequenced mycobacterial and selected actinobacterial species (Continued)

\begin{tabular}{|c|c|c|c|c|c|c|c|c|c|}
\hline M. tuberculosis H37Rv & & $x$ & & $x$ & $x$ & $x$ & $x$ & & \\
\hline M. tusciae JS617 & & $x$ & & $x$ & $x$ & $x^{c}$ & & $x x$ & $x$ \\
\hline M. ulcerans Agy99 & & $x$ & & $x$ & & & $x$ & & \\
\hline M. vaccae ATCC 25954 & & $x$ & $x$ & $x$ & $x$ & & & & \\
\hline M. vanbaalenii PYR-1 & & $x$ & & $x$ & $x$ & & & & \\
\hline M. xenopi RIVM700367 & & $x$ & & $x$ & & $x$ & $x$ & & \\
\hline M. yongonense 05-1390 & & $x$ & & $x$ & & $x$ & $x$ & & \\
\hline Nocardia brasiliensis ATCC700358 & & $x$ & $x$ & & $x^{c}$ & & & & \\
\hline Nocardia cyriacigeorgica GUH-2 & & $x$ & $x$ & & $x^{c}$ & & & & \\
\hline Nocardia farcinica IFM 10152 & & $x$ & $x$ & & & & & & \\
\hline Rhodococcus equi $103 \mathrm{~S}$ & & $x$ & & & & & & & \\
\hline Rhodococcus erythropolis PR4 & & $x$ & & & & & & & \\
\hline Rhodococcus opacus B4 & & $x$ & & & & & & & \\
\hline Staphylococcus aureus USA300 & $x$ & & & & & & & & \\
\hline Streptomyces coelicolor A3 (2) & & $x$ & & & & & & & \\
\hline Saccharopolyspora erythraea NRRL 2338 & & $x x$ & & & & & & & \\
\hline Stackebrandtia nassauensis DSM 44728 & & $x x x x$ & & & & & & & \\
\hline Streptosporangium roseum DSM 43021 & & $x$ & & & & & & & \\
\hline Segniliparus rotundus DSM 44985 & & & $x$ & $x$ & & & & & \\
\hline Salinispora tropica CNB-440 & & $x x$ & & & & & & & \\
\hline Tsukamurella paurometabola DSM 20162 & & & $x$ & & $x^{c}$ & & & & \\
\hline
\end{tabular}

${ }^{\mathrm{a}}$ Sequencing projects are incomplete (as of 07/2015)

${ }^{\mathrm{b}} \mathrm{RD} 1$ deletion within cluster

${ }^{\mathrm{c} A n c e s t r a l}$ region

publicly available and were included in the phylogenetic analyses. The plasmid-encoded ESX clusters group phylogenetically with some of the ESX gene clusters identified on contigs from the incomplete genome sequences of M. tusciae and M. parascrofulaceum and together form a subclade of each genomic ESX duplication subsequent to ESX-4 (Fig. 1). The M. parascrofulaceum and $M$. tusciae sequencing projects are incomplete, therefore it was not possible to conclusively determine whether the ESX gene clusters identified in these species are plasmid or chromosomally located. However, based on synteny and the phylogenetic clustering of these $M$. tusciae and M. parascrofulaceum ESX with the plasmidencoded ESX clusters, these ESX are predicted to be encoded on plasmids, or to originate directly from plasmid DNA. Sequence alignments indicate that each contig containing a predicted plasmid-located ESX cluster shares several conserved segments, or locally collinear blocks (LCBs), with the ESX-containing plasmids from the same subclade (Additional file 3). This is particularly apparent for sequences containing the subclade of ESX3 , which consist almost entirely of four LCBs, and the subclade of ESX-5. This supports the definition of these M. tusciae and M. parascrofulaceum ESX gene clusters as plasmid ESX gene clusters. The ESX gene clusters on the plasmids and M. tusciae and M. parascrofulaceum contigs, which form outgroups to ESX-1, $-2,-3$ and -5 , were named ESX-P1, -P2, -P2', -P3 and -P5, where "P" indicates the plasmid localisation of the ESX (Table 2). ESX-P1, ESX-P2, ESX-P3 and ESX-P5 form outgroups to the genomic ESX with the same numbers and ESX-P2' branches off prior to ESX-P2. ESX-P1 to -P5 contain all of the core ESX components, including esp $\mathrm{G}$ and $e s p \mathrm{I}$ and ESX-P1 also incorporates EspH, while EccA is absent from ESX-P2.

\section{ESX-4}

Orthologs of the ESX-4 gene cluster were identified in all of the mycolic acid producing species from the genera Mycobacterium, Gordonia, Nocardia, Rhodococcus and Corynebacterium. ESX-4 gene clusters were also identified in the 9 species from the orders Pseudonocardiales, Glycomycetales, Micromonosporales, Frankiales, Streptosporangiales, Catenulisporales, Streptomycetales, Propionibacteriales and Kineosporiales which do not have mycolic acids in their cell envelope. These organisms each contain between one and four copies of the ESX-4 gene cluster. Although the arrangement and components of this gene cluster are well conserved amongst the mycobacterial species; insertions, deletions and rearrangements are common amongst the 


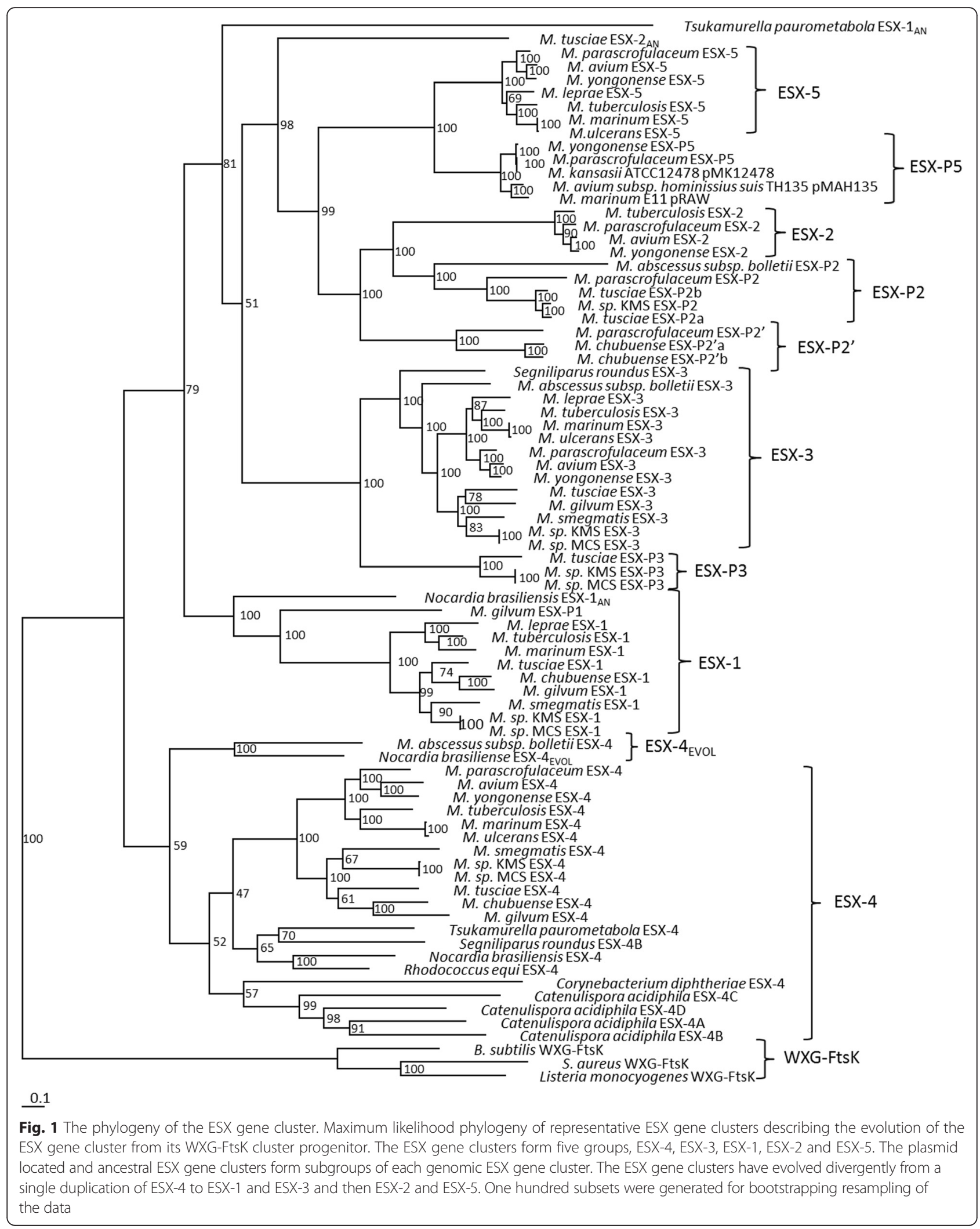


Table 2 The plasmid-encoded ESX clusters

\begin{tabular}{|c|c|c|c|c|}
\hline ESX & Species & Plasmid/contig & Accession number & Size (bp) \\
\hline P1 & M. gilvum PYR-GCK & pMFLV01 & NC_009339.1 & 321,253 \\
\hline \multirow[t]{5}{*}{ P2 } & M. abscessus subsp. bolletii 50594 & plasmid 2 & NC_021279.1 & 97,240 \\
\hline & M. parascrofulaceum BAA-614 & contig00115 & ADNV01000102.1 & 21,921 \\
\hline & M. sp KMS & pMKMS01 & NC_008703.1 & 302,089 \\
\hline & M. tusciae JS617 & contig 196 & NZ_AGJJ01000027.1 & 108,484 \\
\hline & M. tusciae JS617 & contig 209 & NZ_AGJJ01000007.1 & 249,244 \\
\hline \multirow[t]{3}{*}{$P 2^{\prime}$} & M. chubuense & pMYCCH.01 & NC_018022 & 615,278 \\
\hline & M. chubuense & pMYCCH.02 & NC_018023 & 143,623 \\
\hline & M. parascrofulaceum BAA-614 & contig00017 & ADNV01000015.1 & 70,331 \\
\hline \multirow[t]{3}{*}{ P3 } & M. sp KMS & pMKMSO2 & NC_008704.1 & 216,763 \\
\hline & M. sp. MCS & Plasmid1 & NC_008147.1 & 215,075 \\
\hline & M. tusciae JS617 & contig 224 & NZ_AGJJ01000010.1 & 218,303 \\
\hline \multirow[t]{5}{*}{ P5 } & M. avium subsp. hominissuis suis $\mathrm{TH} 135$ & pMAH135 & AP012556 & 194,711 \\
\hline & M. kansasii ATCC12478 & pMK12478 & СР006836 & 144,951 \\
\hline & M. marinum E11 & pRAW & HG917973 & 114,229 \\
\hline & M. parascrofulaceum BAA-614 & contig00109 & ADNV01000096.1 & 47,725 \\
\hline & M. yongonense 05-1390 & pMyong1 & JQ657805 & 122,976 \\
\hline
\end{tabular}

non-Mycolata. The ESX-4 gene cluster contains genes encoding the FtsK/SpoIIIE protein EccC, and two WXG proteins, EsxU and EsxT, which are present in the FtsK-WXG clusters of $S$. aureus, L. monocytogenes and B. subtilis. In addition to the WXG-FtsK cluster components, ESX-4 encodes EccD, EccB and MycP, which have been suggested to be involved in a more intricate secretion mechanism to transport proteins into and across the unique and complex outer mycomembrane [34]. However, the presence of the ESX-4 cluster in various non-mycomembrane containing actinobacteria suggests that the secretion system encoded by these gene clusters is not directly involved in mycomembrane translocation. Although the function(s) of ESX-4 have yet to be determined, the presence and maintenance of this gene cluster throughout the mycobacteria and other actinobacteria suggests that it plays an important role in bacterial metabolism. Homologs of the ESX-4 gene cluster components occur in all 5 ESX gene clusters and could represent the proteins required for translocation across the inner membrane. The additional components present in the subsequent ESX duplications may be involved in mycomembrane translocation, be additional substrates, assist in the translocation of additional substrates or facilitate specific mechanisms of those secretion systems.

Phylogenetically associated with the ESX-4 gene cluster is a subgroup of ESX gene clusters which include homologs of the eccA, eccE, esp $\mathrm{G}$, esp I, pe and ppe genes, in addition to the ESX-4 components. This cluster was identified in the mycolic acid producing species $N$. farcinica, N. brasiliense, N. cyriacigeorgica, T. paurometabola, S. rotundus, M. vaccae, $M$. fortuitum and $M$. abscessus subsp. bolletii. The arrangement of the genes in this cluster varies between species, but does not resemble any of the $M$. tuberculosis ESX gene clusters. This cluster contains all of the conserved ESX gene cluster components and appears to be an evolutionary intermediate between ESX-4 and the subsequent duplications, and is therefore named ESX-4 EVOL (ESX-4 evolved).

\section{ESX-3}

ESX-3 present in all of the studied mycobacteria, with the exception of $M$. chubuense, suggesting that ESX-3 is the first ESX duplication in the mycobacterial genome. ESX-3 contains all of the ESX conserved components eccA to $\mathrm{E}, m y c \mathrm{P}$, esx and pe/ppe pairs as well as esp G. Although essential for in vitro growth of $M$. tuberculosis, ESX-3 is not essential in the fast-growing M. smegmatis [20]. ESX-3 is involved in iron homeostasis and uptake via the mycobactin pathway [24] and genetic reduction during evolution of the slowgrowers may have eliminated the redundancy of ESX3. Outside of the mycobacteria, ESX-3 was only identified in $S$. rotundus suggesting that ESX-3 was inserted prior to the divergence of Segniliparus and Mycobacterium from a common ancestor. The presence of three mycobactin genes in the $S$. rotundus ESX-3 furthermore suggests that the association between ESX-3 and iron homeostasis may be conserved. The ancestral mycobacteria $M$. abscessus, M. abscessus subsp. bolletii and $M$. massiliense contain only ESX-4 (or ESX-4evol) and ESX-3. 


\section{ESX-1}

ESX-1 is present in all of the other fast-growing mycobacteria; $M$. thermoresistibile, $M$. smegmatis $\mathrm{mc}^{2} 155, M$. neoaurum, $M$. fortuitum, M. vanbaalenii, M. gilvum, $M$. sp. Spyr1, M. vaccae M. rhodesiae, M. phlei, M. sp. JLS, M. sp. KMS and M. sp. MCS; but has been deleted from the genomes of various slow-growing mycobacteria ( $M$. avium, M. avium paratuberculosis, M. colombiense, M. intracellulare, M. parascrofulaceum, M. ulcerans, M. xenopi, $M$. indicus pranii, M. sp. MOTT36Y and M. sp. JDM601), with partial deletions (Region of Deletion 1, RD1) in M. bovis BCG and M. microti. ESX-1 contains both esp G and espI, and in most cases eccE and $m y c \mathrm{P}$ have been inverted along with the insertion of several additional genes. ESX-1 has been implicated in virulence, and its deletion in attenuation of the pathogenic mycobacteria [8, 9]. However, its presence throughout most of the mycobacteria, including non-pathogenic and saprophytic fast-growing organisms, suggests that the primary function of this gene cluster is not virulence, and that the virulence-associated function has evolved more recently in pathogenic organisms.

An additional gene cluster, identified in the nonmycobacterial actinomycetes $N$. brasiliense and N. cyriacigeorgica contains all of the components of ESX-4 $4_{\mathrm{EVOL}}$, but has an operonic arrangement similar to the $M$. tuberculosis ESX gene clusters. This cluster forms a subgroup just outside of the mycobacterial ESX-1 clade and is therefore named ESX-1 $1_{\mathrm{AN}}$ (ancestral ESX-1). An ESX gene cluster with similar arrangements was identified in T. paurometabola, but has undergone a transposition event which has resulted in the disruption of $e c c \mathrm{C}$ and deletion of eccB. Phylogenetic clustering of this region is not consistent between algorithms and this region is also predicted to be an ESX-1 $1_{\mathrm{AN}}$ cluster, based on synteny.

\section{ESX-2 and ESX-5}

ESX-2 and ESX-5 occur only in the slow-growing mycobacteria. ESX-2 contains all of the conserved ESX components including esp $\mathrm{G}$ and espI in an operonic structure, while ESX-5 contains only esp G, but has multiple copies of pe and ppe, and the insertion of a ferredoxin and a cyp 143 gene. The function(s) of ESX-2 have not been elucidated, and although its duplication correlates evolutionarily with both the slow-growing and pathogenic phenotypes, it has been lost from some of these species (M. leprae, M. marinum, M. ulcerans subsp. liflandii and M. ulcerans). ESX-5 is the only ESX gene cluster present in all of the slow-growers but absent in all of the fast-growers, and may be the ESX gene cluster most involved in pathogenicity and the slowgrowing phenotype [35]. Deletion of this region, however, does not directly increase the growth rate of $M$. marinum or $M$. tuberculosis $[18,36]$. ESX-5 has been implicated in immune evasion and in the secretion of the PE and PPE proteins [36, 37]. Only ESX-5 contains multiple copies of the pe and ppe genes, the numbers of which vary between species, and its evolution is predicted to have preceded the expansion of these gene families [37].

M. tusciae contains an ESX cluster, ESX-2 $2_{\mathrm{AN}}$ (ancestral ESX-2), which contains all of the ESX-2 components and precedes both the ESX-2 and ESX-5 clades, as well as ESX-P2', -P2 and -P5 gene clusters. M. tusciae is a slow-growing mycobacterium which, based on $16 \mathrm{~S}$ rDNA sequencing, clusters with the fast-growing mycobacteria and is most closely related to the fast-growing mycobacteria $M$. farcinogenes, $M$. komossense and $M$. aichiense [38]. The correlation between the presence of an ESX-2/5-like cluster and a slow growth-rate might imply that $M$. tusciae is an evolutionary intermediate between the fast- and slow-growing mycobacteria. The mycolic acid composition of the cell membrane of $M$. tusciae most closely resembles that of the M. avium complex and M. parascofulaceum [38] suggesting that the different ESX secretion systems may have evolved with changes in the mycomembrane structure; as reflected in the role of ESX-5 in maintaining selective mycomembrane permeability in the slow growing pathogenic $M$. tuberculosis and M. marinum species [19]. Investigation of the potential association between these two ESX clusters, mycomembrane structure and growth rate may provide important information regarding the evolution of the often pathogenic, slow-growing mycobacteria.

\section{Plasmid-mediated ESX evolution}

The duplication and evolution of the ESX gene clusters and their secretion systems have clearly impacted on the evolution, diversity and success of the mycobacteria. The identification of ESX gene clusters on several plasmids within the mycobacteria, and their phylogenetic association with each of the genomic ESX gene clusters, provides novel insight into the mechanism of ESX evolution suggesting that the duplication and diversification of these clusters was plasmid-mediated. The presence of multiple plasmid copies within a single organism facilitates diversification by allowing the coevolution of various ESX clusters simultaneously. The plasmid localisation furthermore facilitates the loss of deleterious effects, while the incorporation of beneficial plasmid DNA into the genome allows permanent retention and might be selected for. We propose a model for the plasmidmediated duplication and evolution of the ESX gene clusters (Fig. 2).

Based on this model, the FtsK-WXG cluster present in the Firmicutes evolved to form the ESX-4 gene cluster, through the incorporation of $e c c \mathrm{~B}, e c c \mathrm{D}$ and $m y c \mathrm{P}$, during the evolution of the actinobacteria; resulting in the presence of ESX-4 in the genomes of various actinobacterial 


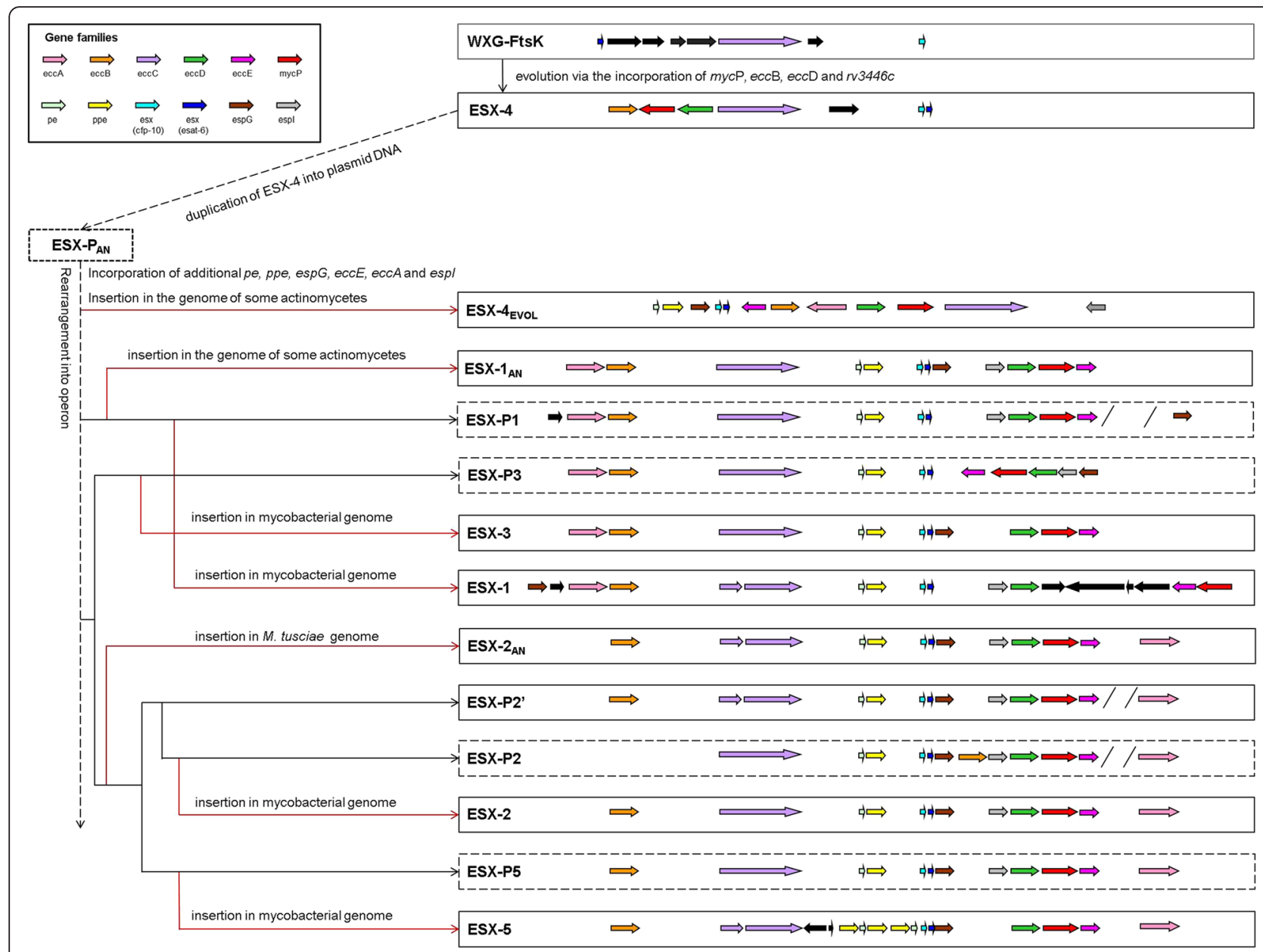

Fig. 2 Model of ESX evolution based on plasmid-mediated duplication and evolution. The ancestral ESX-4 gene cluster evolved from the WXGFtsK cluster via the incorporation of additional genes, eccB, eccD, mycP and rv3446c. ESX-4 was duplicated into plasmid DNA, into which additional ESX genes, eccA, eccE, espl, espG, pe and ppe, were incorporated. The plasmid ancestor (ESX-PAN) was reinserted into the genomes of various

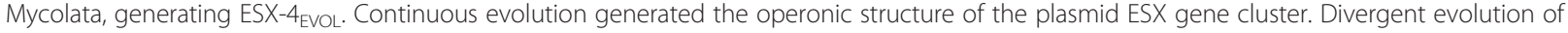
the plasmid ESX generated several plasmid ESX (ESX-P1, -P3, -P2', -P2 and -P5) which were inserted into the mycobacterial genome to generate ESX-3, ESX-1, ESX-2 and ESX-5. An earlier version of ESX-P1 was inserted into the genomes of some actinomycetes as ESX-1 AN $_{\text {and }}$ a precursor of ESX-P2' was inserted into the M. tusciae genome as ESX-2AN. Red arrows represent genome insertions

species. A copy of ESX-4 has been incorporated into plasmid DNA after the divergence of the genera Corynebacterium and Rhodococcus. The additional ESX components, eccA, eccE, espG, espI, pe and ppe, were incorporated into this plasmid-located cluster $\left(E S X-P_{A N}\right)$, which was subsequently incorporated into the genomes of some species, including Nocardia ssp., T. paurometabola, S. rotundus and $M$. abscessus subsp. bolletii, as ESX- $4_{\mathrm{EVOL}}$. The variation in the arrangement and sequences of the genes in these clusters may represent independent insertions at different evolutionary time points. The presence of both ESX-4 and ESX-4 $4_{\mathrm{EVOL}}$ in some species implies that ESX$4_{\mathrm{EVOL}}$ is a duplication of the ESX-4 cluster, and has not evolved directly from it. ESX-1, $-2,-3$, and 5 have evolved from a single duplication of ESX-4. The presence of all of the conserved ESX components in ESX-4 $4_{\mathrm{EVOL}}$ suggests that it evolved from the same progenitor and that ESX$4_{\mathrm{EVOL}}$ is an intermediate between ESX-4 and ESX-1, -2, -3 and -5 . Continual evolution of this plasmid ESX gene cluster generated the operonic structure characteristic of the mycobacterial ESX gene cluster duplications. Plasmid precursors of the four duplications, ESX-P1, -P3, -P2', -P2 and -P5, have evolved simultaneously by divergence of the common plasmid ancestor, after which genome insertions generated the genomic ESX-1, $-2,-3$ and -5 clusters.

It appears furthermore, that these plasmids may be able to transfer between mycobacterial species. The pRAW, pMyong1, pMK12478 and pMAH135 plasmids, which contain ESX-P5, were also shown to contain components of a Type-IV secretions system and a traA/relaxase gene; which are required for conjugation of the plasmid between some slow-growing mycobacterial species [33]. 


\section{ESX-associated evolution of the mycobacteria}

A phylogenetic analysis of the mycobacteria and related actinomycetes based on their ESX gene clusters was done using the concatenated protein sequences of all of the ESX gene clusters of each species (Fig. 3). The Mycolata have evolved from a single gram-positive monoderm

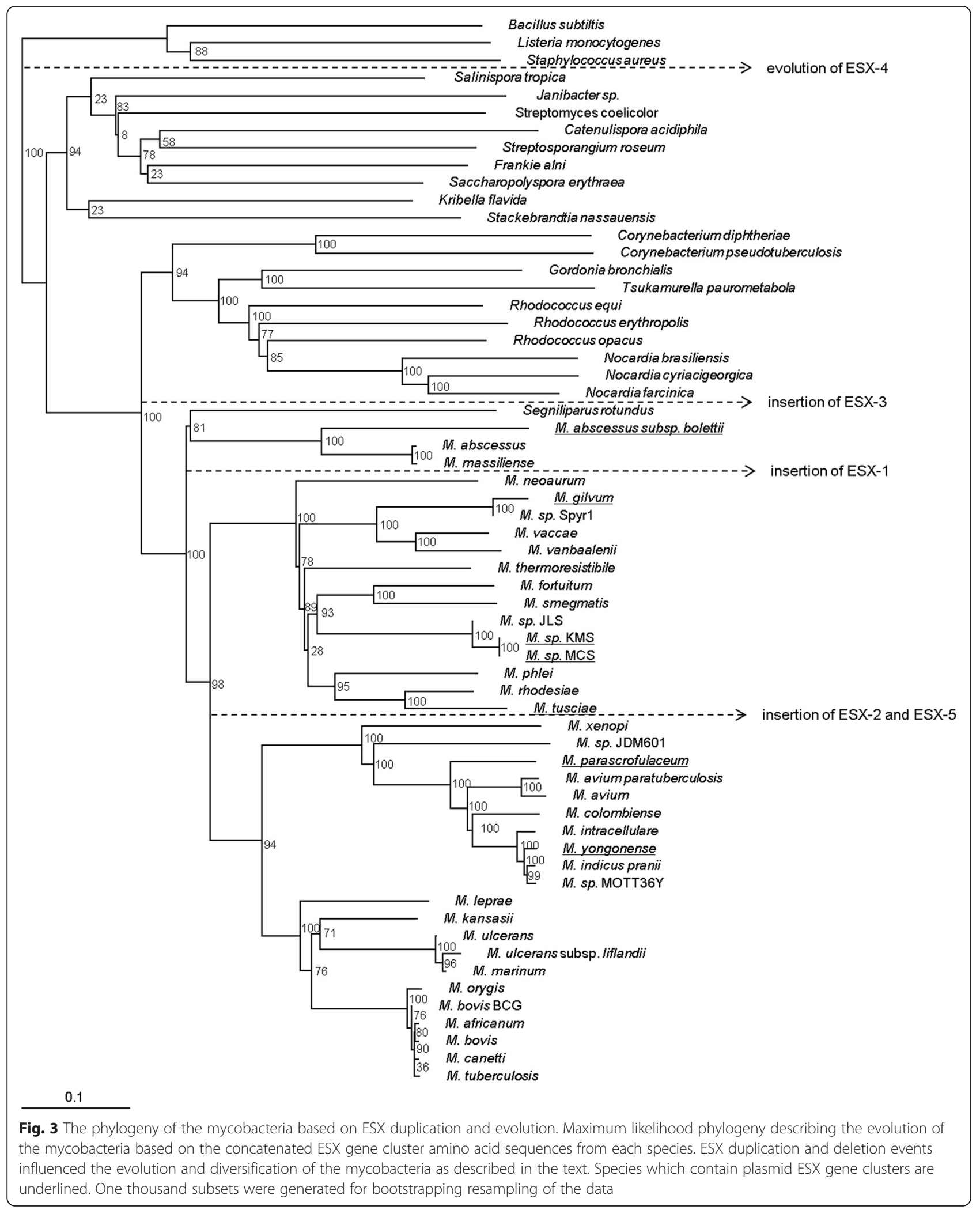


ancestor into two groups, those which contain only ESX4, ESX- $4_{\mathrm{EVOL}}$ and $\mathrm{ESX}-1_{\mathrm{AN}}$, the non-mycobacterial actinomycetes; and those which also contain an ESX-3 gene cluster, which with the exception of $S$. rotundus, consist of the mycobacteria. $S$. rotundus contains ESX-4 $4_{\mathrm{EVOL}}$ and ESX-3, while all of the mycobacteria contain at least ESX4 and ESX-3, with the exception of $M$. leprae which has lost ESX-4. ESX-1 was incorporated in the mycobacterial genome after the divergence of $M$. abscessus and M. massiliense, and is present in all of the other fast-growing mycobacteria. However, an ESX-1-like cluster $\left(\right.$ ESX-1 $\left.1_{\mathrm{AN}}\right)$ is also present in some Nocardia ssp.. ESX-1 $1_{\mathrm{AN}}$ predates ESX-P1 and was likely incorporated into the genome from an earlier form of ESX-P1, after the divergence of the mycobacteria. The presence of ESX-1 $1_{\mathrm{AN}}$ in the absence of ESX-3 in some Nocardia species, and the presence of ESX-3 in the absence of ESX-1 in $M$. abscessus ssp., $M$. massiliense and $S$. rotundus suggests that these plasmid clusters evolved simultaneously in an ancestral species, and were inserted into the genomes of the different organisms at different times. The role of ESX-1 in conjugal DNA transfer in M. smegmatis $[25,26]$ may be linked to its origin in plasmid DNA, where it may have facilitated the transfer of the plasmid during cell division.

The presence of ESX-2 and ESX-5 marks the emergence of the slow-growing mycobacteria. ESX-2 and ESX-5 evolved from a common ancestral plasmid-ESX, which diverged to produce ESX-P2' and ESX-P2; and -P5. ESX-2 and ESX-5 were integrated into the mycobacterial genome with the divergence of the slow-growing mycobacteria, however the presence of ESX-2 ${ }_{\mathrm{AN}}$, ESX-P2' and ESX-P2 in various fast-growing mycobacteria attests to the presence of these precursors earlier in mycobacterial evolution. The $M$. avium complex can be distinguished by the transposition of $\mathrm{EccB}_{2}$ and $\mathrm{EccC}_{2}$. ESX-2 was deleted from a precursor of $M$. ulcerans, $M$. ulcerans subsp. liflandii and $M$. marinum. ESX-1 was deleted from the genomes of slowgrowing mycobacteria on numerous occasions. M. kansasii and the $M$. tuberculosis complex have retained all five ESX gene clusters, with the exception of $M$. bovis BCG and $M$. microti (not shown), which contain the previously described RD1 deletions in ESX-1 [3, 4, 39-41]. M. leprae, which has undergone extensive gene reduction, has retained only ESX-3, -1 and -5 and $M$. ulcerans has retained only ESX-4, -3 and -5 .

\section{Conclusion}

The distinctive cell envelope of mycobacteria, characterised by the highly impermeable outer mycomembrane peptidoglycan-arabinogalactan-mycolic acid matrix [6], provides a protective barrier against extracellular stresses, but also presents an obstacle to the export of proteins and acquisition of nutrients. Although mycobacteria possess both Sec and Tat secretion systems, which translocate proteins across the inner membrane, the ESX, or Type VII, secretion systems are the first mechanism proposed for the secretion of proteins into and across the mycomembrane. This study explored the evolution of the mycobacterial Type VII ESX gene clusters from the WXG-FtsK cluster in $S$. aureus, $L$. monocytogenes and $B$. subtilis to the 5 ESX gene clusters in $M$. tuberculosis. The ancestral ESX gene cluster (ESX-4) was identified in several non-mycomembrane producing actinobacteria as well as the non-mycobacterial Corynebacteriales. Between two and seven ESX gene clusters were identified in each mycobacterial species. A novel ESX gene cluster, ESX-4 $4_{\mathrm{EVOL}}$, was identified in some non-mycobacterial myco-membrane containing actinomycetes and $M$. abscessus subsp. bolletii. ESX-4 $4_{\mathrm{EVOL}}$ contains all of the conserved components of the ESX and appears to be a precursor of the mycobacterial ESX duplications. Plasmid-encoded precursor ESX were identified for each of the genomic ESX-3, -1, -2 and -5 gene clusters and a novel plasmid-mediated mechanism of ESX duplication and evolution proposed. The presence and absence of the ESX gene clusters in the mycobacteria redefines the order of duplication of the ESX gene clusters in the mycobacteria as ESX-4, ESX-3, ESX-1 and then ESX-2 and ESX-5. The influence of the various ESX gene clusters on vital biological and virulence-related functions has clearly influenced the diversification and success of the various mycobacteria, and their evolution from the non-pathogenic fast-growing saprophytic to the slowgrowing pathogenic organisms.

\section{Methods}

\section{Genome sequence data}

All protein and DNA sequence information was obtained from publicly available finished and unfinished genome sequencing information. The genomes of 40 mycobacterial species, 11 other species from the order Corynebacteriale, nine species selected from the orders Pseudonocardiales, Glycomycetales, Frankiales, Micromonosporales, Streptosporangiales, Catenulisporales, Streptomycetales, Propionibacteriales and Kineosporiales and 3 gram-positive monoderm species containing WXG-FtsK clusters (Table 1), were analysed.

\section{Comparative genomic analyses}

The M. tuberculosis H37Rv ESX protein sequences of interest were used as templates to identify orthologous ESX protein and gene sequences. Blast similarity searches, blastn, tblastn and blastp [42], were done using NCBI Blast and the genome sequence databases listed in Additional file 4. Adjacent genomic regions were searched for additional ESX genes to determine clustering and arrangement of genes; for unfinished genomes 
in contig format this was not always possible and gene cluster arrangement was assumed based on sequence identity and anticipated arrangement. Large intergenic regions were searched for gene insertions using blastx analyses [43].

\section{Phylogenetic analyses}

Annotated protein sequences were obtained from the protein sequence databases. The protein sequences of conserved components of each ESX gene cluster (EccA, EccB, EccC, EccD, EccE, PE(s), PPE(s), Esx (CFP-10like), Esx (ESAT-6-like), EspG, EspI, MycP, Rv3446c, EspH, EspJ, EspK, EspL, EspB, Cyp143 and Ferredoxin) were concatenated. Multiple sequence alignments of all concatenated ESX gene cluster protein sequences were done with Clustal W $2.0[44,45]$ using the Bioedit Sequence Alignment Editor version 7.1.3.0 [46]. Similarly, multiple sequence alignments of a single sequence composed of all of the combined ESX gene cluster protein sequences, from each species, were done. Phylogenetic trees were determined by distance and maximum likelihood analyses using SeaView Version 4.4.2 [47]. Distance analysis was done using the observed neighbour-joining method with 10000 bootstrap replicates. Maximum likelihood phylogenies were generated using PhyML [48] with the JTT (Jones Taylor Thornton) algorithm [49], using model-given amino acid equilibrium frequencies, specifying no invariable sites and no across site variation. Nearest-neighbor interchange tree searching operations were used with a BioNJ starting tree. The WXG-FtsK cluster sequences from $S$. aureus, L. monocytogenes and $B$. subtilis were defined as the outgroup. The M. microti ESX clusters were omitted from the phylogenetic analyses as protein annotations were not available.

\section{Plasmid and contig sequence alignments}

Plasmid and contig sequences were obtained from the NCBI (Additional file 4) and alignments of the plasmid and contig sequences containing each subgroup of ESX gene cluster were done using the progressiveMauve algorithm of the Mauve 2.3.1 Genome Alignment Visualisation software [50].

\section{Availability of supporting data}

All supporting data are included as Additional files 1, 2, 3 and 4.

\section{Additional files}

Additional file 1: The ESX gene clusters of sequenced mycobacteria and selected actinobacterial species. (DOCX $3463 \mathrm{~kb}$ )

Additional file 2: Neighbour joining phylogeny of the ESX gene clusters using 10000 bootstrap replicates. (PNG 33 kb)
Additional file 3: Mauve sequence alignments of ESX-P containing plasmids and contigs which are predicted to be plasmids.

Additional file 4: Analysed genomes. (XLSX 20 kb)

Competing interests

The authors declare that they have no competing interests.

\section{Authors' contributions}

MNF participated in the design of the study, carried out the analyses and wrote the initial draft of the manuscript. RMW, SLS and PDVH participated in the design of the study and advised on computational methodologies and the interpretation of data. NGVP conceived of and designed the study and advised on computational methodologies and the interpretation of data. All authors contributed to, read and approved the final manuscript.

Received: 30 November 2015 Accepted: 6 March 2016

Published online: 15 March 2016

References

1. Tekaia F, Gordon SV, Garnier T, Brosch R, Barrell BG, Cole ST. Analysis of the proteome of Mycobacterium tuberculosis in silico. Tuber Lung Dis. 1999;79: 329-42.

2. Gey Van Pittius NC, Gamieldien J, Hide W, Brown GD, Siezen RJ, Beyers AD. The ESAT-6 gene cluster of Mycobacterium tuberculosis and other high G+C Gram-positive bacteria. Genome Biol. 2001;2:RESEARCH0044.

3. Mahairas GG, Sabo PJ, Hickey MJ, Singh DC, Stover CK. Molecular analysis of genetic differences between Mycobacterium bovis BCG and virulent M. bovis. J Bacteriol. 1996:178:1274-82.

4. Behr MA, Wilson MA, Gill WP, Salamon H, Schoolnik GK, Rane S, Small PM. Comparative genomics of BCG vaccines by whole-genome DNA microarray. Science. 1999;284:1520-3.

5. Brosch R, Gordon SV, Buchrieser C, Pym AS, Garnier T, Cole ST. Comparative genomics uncovers large tandem chromosomal duplications in Mycobacterium bovis BCG Pasteur. Yeast. 2000;17:111-23.

6. Abdallah AM, Gey van Pittius NC, Champion PA, Cox J, Luirink J, Vandenbroucke-Grauls CM, Appelmelk BJ, Bitter W. Type VII secretionmycobacteria show the way. Nat Rev. 2007;5:883-91.

7. Bitter W, Houben EN, Bottai D, Brodin P, Brown EJ, Cox JS, Derbyshire K, Fortune SM, Gao LY, Liu J, Gey van Pittius NC, Pym AS, Rubin EJ, Sherman DR, Cole ST, Brosch R. Systematic genetic nomenclature for type VII secretion systems. PLoS Pathog. 2009:5:e1000507.

8. Hsu T, Hingley-Wilson SM, Chen B, Chen M, Dai AZ, Morin PM, Marks CB, Padiyar J, Goulding C, Gingery M, Eisenberg D, Russell RG, Derrick SC, Collins FM, Morris SL, King CH, Jacobs Jr WR. The primary mechanism of attenuation of bacillus Calmette-Guerin is a loss of secreted lytic function required for invasion of lung interstitial tissue. Proc Natl Acad Sci U S A. 2003;100:12420-5.

9. Lewis KN, Liao R, Guinn KM, Hickey MJ, Smith S, Behr MA, Sherman DR. Deletion of RD1 from Mycobacterium tuberculosis mimics bacille CalmetteGuerin attenuation. J Infect Dis. 2003;187:117-23.

10. Pym AS, Brodin P, Majlessi L, Brosch R, Demangel C, Williams A, Griffiths KE, Marchal G, Leclerc C, Cole ST. Recombinant BCG exporting ESAT-6 confers enhanced protection against tuberculosis. Nat Med. 2003;9:533-9.

11. MacGurn JA, Cox JS. A genetic screen for Mycobacterium tuberculosis mutants defective for phagosome maturation arrest identifies components of the ESX-1 secretion system. Infect Immun. 2007:75:2668-78.

12. Samten B, Wang X, Barnes PF. Mycobacterium tuberculosis ESX-1 systemsecreted protein ESAT- 6 but not CFP10 inhibits human T-cell immune responses. Tuberculosis (Edinb). 2009;89 Suppl 1:S74-6.

13. de Jonge MI, Pehau-Arnaudet G, Fretz MM, Romain F, Bottai D, Brodin P, Honore N, Marchal G, Jiskoot W, England P, Cole ST, Brosch R. ESAT-6 from Mycobacterium tuberculosis dissociates from its putative chaperone CFP-10 under acidic conditions and exhibits membrane-lysing activity. J Bacteriol. 2007;189:6028-34.

14. Smith J, Manoranjan J, Pan M, Bohsali A, Xu J, Liu J, McDonald KL, Szyk A, LaRonde-LeBlanc N, Gao LY. Evidence for pore formation in host cell membranes by ESX-1-secreted ESAT-6 and its role in Mycobacterium marinum escape from the vacuole. Infect Immun. 2008;76:5478-87.

15. Abdallah AM, Bestebroer J, Savage ND, de Punder K, van Zon M, Wilson L, Korbee CJ, van der Sar AM, Ottenhoff TH, van der Wel NN, Bitter W, Peters PJ. 
Mycobacterial secretion systems ESX-1 and ESX-5 play distinct roles in host cell death and inflammasome activation. J Immunol. 2011;187:4744-53.

16. Houben D, Demangel C, van Ingen J, Perez J, Baldeon L, Abdallah AM, Caleechurn L, Bottai D, van Zon M, de Punder K, van der Laan T, Kant A, Bossers-de Vries R, Willemsen P, Bitter W, van Soolingen D, Brosch R, van der Wel N, Peters PJ. ESX-1-mediated translocation to the cytosol controls virulence of mycobacteria. Cell Microbiol. 2012;4:1287-98.

17. Abdallah AM, Verboom T, Hannes F, Safi M, Strong M, Eisenberg D, Musters RJ, Vandenbroucke-Grauls CM, Appelmelk BJ, Luirink J, Bitter W. A specific secretion system mediates PPE41 transport in pathogenic mycobacteria. Mol Microbiol. 2006;62:667-79.

18. Weerdenburg EM, Abdallah AM, Mitra S, de Punder K, van der Wel NN, Bird S, Appelmelk BJ, Bitter W, van der Sar AM. ESX-5-deficient Mycobacterium marinum is hypervirulent in adult zebrafish. Cell Microbiol. 2012;14:728-39.

19. Ates $L S$, Ummels $R$, Commandeur $S$, van der Weerd $R$, Sparrius $M$, Weerdenburg E, Alber M, Kalscheuer R, Piersma SR, Abdallah AM, Abd El Ghany M, Abdel-Haleem AM, Pain A, Jiménez CR, Bitter W, Houben ENG. Essential Role of the ESX-5 Secretion System in Outer Membrane Permeability of Pathogenic Mycobacteria. PLoS Genet. 2015;11:e1005190.

20. Sassetti CM, Boyd DH, Rubin EJ. Genes required for mycobacterial growth defined by high density mutagenesis. Mol Microbiol. 2003;48:77-84.

21. Serafini A, Boldrin F, Palu G, Manganelli R. Characterization of a Mycobacterium tuberculosis ESX-3 conditional mutant: essentiality and rescue by iron and zinc. J Bacteriol. 2009;191:6340-4.

22. Maciag A, Dainese E, Rodriguez GM, Milano A, Prowedi R, Pasca MR, Smith I, Palù G, Riccardi G, Manganelli R. Global analysis of the Mycobacterium tuberculosis Zur (FurB) regulon. J Bacteriol. 2007;189:730-40.

23. Rodriguez GM, Voskuil MI, Gold B, Schoolnik GK, Smith I. ideR, An essential gene in Mycobacterium tuberculosis: role of IdeR in iron-dependent gene expression, iron metabolism, and oxidative stress response. Infect Immun. 2002;70:3371-81.

24. Siegrist MS, Unnikrishnan M, McConnell MJ, Borowsky M, Cheng TY, Siddiqi N, Fortune SM, Moody DB, Rubin EJ. Mycobacterial Esx-3 is required for mycobactin-mediated iron acquisition. Proc Natl Acad Sci U S A. 2009;106: 18792-7.

25. Flint JL, Kowalski JC, Karnati PK, Derbyshire KM. The RD1 virulence locus of Mycobacterium tuberculosis regulates DNA transfer in Mycobacterium smegmatis. Proc Natl Acad Sci U S A. 2004;101:12598-603.

26. Coros A, Callahan B, Battaglioli E, Derbyshire KM. The specialized secretory apparatus ESX-1 is essential for DNA transfer in Mycobacterium smegmatis. Mol Microbiol. 2008;69:794-808.

27. Maciag A, Piazza A, Riccardi G, Milano A. Transcriptional analysis of ESAT-6 cluster 3 in Mycobacterium smegmatis. BMC Microbiol. 2009;9:48.

28. Wirth SE, Krywy JA, Aldridge BB, Fortune SM, Fernandez-Suarez M, Gray TA, Derbyshire KM. Polar assembly and scaffolding proteins of the virulenceassociated ESX-1 secretory apparatus in mycobacteria. Mol Microbiol. 2012; 83:654-64.

29. Converse SE, Cox JS. A protein secretion pathway critical for Mycobacterium tuberculosis virulence is conserved and functional in Mycobacterium smegmatis. J Bacteriol. 2005;187:1238-45.

30. Pallen MJ. The ESAT-6/WXG100 superfamily - and a new Gram-positive secretion system? Trends Microbiol. 2002;10:209-12.

31. Sutcliffe IC. New insights into the distribution of WXG100 protein secretion systems. Antonie Van Leeuwenhoek. 2011;99:127-31.

32. Desvaux M, Hebraud M, Talon R, Henderson IR. Outer membrane translocation: numerical protein secretion nomenclature in question in mycobacteria. Trends Microbiol. 2009;17:338-40.

33. Ummels R, Abdallah AM, Kuiper V, Aâjoud A, Sparrius M, Naeem R, Spaink HP, van Soolingen D, Pain A, Bitter W. Identification of a novel conjugative plasmid in mycobacteria that requires both type IV and type VII secretion. MBio. 2014;5: e01744-14.

34. Brodin P, Majlessi L, Marsollier L, de Jonge MI, Bottai D, Demangel C, Hinds J, Neyrolles O, Butcher PD, Leclerc C, Cole ST, Brosch R. Dissection of ESAT-6 system 1 of Mycobacterium tuberculosis and impact on immunogenicity and virulence. Infect Immun. 2006;74:88-98.

35. Abdallah AM, Savage ND, van Zon M, Wilson L, Vandenbroucke-Grauls CM, van der Wel NN, Ottenhoff TH, Bitter W. The ESX-5 secretion system of Mycobacterium marinum modulates the macrophage response. J Immunol. 2008;181:7166-75.

36. Bottai D, Di Luca M, Majlessi L, Frigui W, Simeone R, Sayes F, Bitter W, Brennan MJ, Leclerc C, Batoni G, Campa M, Brosch R, Esin S. Disruption of the ESX-5 system of Mycobacterium tuberculosis causes loss of PPE protein secretion, reduction of cell wall integrity and strong attenuation. Mol Microbiol. 2012;83:1195-209.

37. van Pittius NC G, Sampson SL, Lee H, Kim Y, van Helden PD, Warren RM. Evolution and expansion of the Mycobacterium tuberculosis PE and PPE multigene families and their association with the duplication of the ESAT-6 (esx) gene cluster regions. BMC Evol Biol. 2006;6:95.

38. Tortoli E, Kroppenstedt RM, Bartoloni A, Caroli G, Jan I, Pawlowski J, Emler S. Mycobacterium tusciae sp. nov. Int J Syst Bacteriol. 1999;49 Pt 4:1839-44.

39. van Soolingen D, van der Zanden AG, de Haas PE, Noordhoek GT, Kiers A, Foudraine NA, Portaels F, Kolk AH, Kremer K, van Embden JD. Diagnosis of Mycobacterium microti infections among humans by using novel genetic markers. J Clin Microbiol. 1998;36:1840-5.

40. Brodin P, Eiglmeier K, Marmiesse M, Billault A, Garnier T, Niemann S, Cole ST, Brosch R. Bacterial artificial chromosome-based comparative genomic analysis identifies Mycobacterium microti as a natural ESAT-6 deletion mutant. Infect Immun. 2002;70:5568-78.

41. Gordon SV, Brosch R, Billault A, Garnier T, Eiglmeier K, Cole ST. Identification of variable regions in the genomes of tubercle bacilli using bacterial artificial chromosome arrays. Mol Microbiol. 1999:32:643-55.

42. Altschul SF, Gish W, Miller W, Myers EW, Lipman DJ. Basic local alignment search tool. J Mol Biol. 1990;215:403-10.

43. Gish W, States DJ. Identification of protein coding regions by database similarity search. Nat Genet. 1993;3:266-72.

44. Thompson JD, Higgins DG, Gibson TJ. CLUSTAL W: improving the sensitivity of progressive multiple sequence alignment through sequence weighting, position-specific gap penalties and weight matrix choice. Nucleic Acids Res. 1994;22:4673-80.

45. Larkin MA, Blackshields G, Brown NP, Chenna R, McGettigan PA, McWilliam H, Valentin F, Wallace IM, Wilm A, Lopez R, Thompson JD, Gibson TJ, Higgins DG. Clustal W and Clustal X version 2.0. Bioinformatics. 2007;23:2947-8.

46. Hall TA. BioEdit: a user-friendly biological sequence alignment editor and analysis program for Windows 95/98/NT. Nucl Acids Symp Ser. 1999;41:95-8.

47. Gouy M, Guindon S, Gascuel O. SeaView version 4: A multiplatform graphical user interface for sequence alignment and phylogenetic tree building. Mol Biol Evol. 2010;27:221-4.

48. Guindon S, Delsuc F, Dufayard JF, Gascuel O. Estimating Maximum Likelihood Phylogenies with PhyML. Volume 537. 2009.

49. Jones DT, Taylor WR, Thornton JM. The rapid generation of mutation data matrices from protein sequences. Comput Appl Biosci. 1992;8:275-82.

50. Darling ACE, Mau B, Blattner FR, Perna NT. Mauve: multiple alignment of conserved genomic sequence with rearrangements. Genome Res. 2004;14: 1394-403.

\section{Submit your next manuscript to BioMed Central and we will help you at every step:}

- We accept pre-submission inquiries

- Our selector tool helps you to find the most relevant journal

- We provide round the clock customer support

- Convenient online submission

- Thorough peer review

- Inclusion in PubMed and all major indexing services

- Maximum visibility for your research

Submit your manuscript at www.biomedcentral.com/submit
Biomed Central 